\title{
STRICTLY CONVEX NORMED LINEAR SPACES
}

\author{
S. GUDDER AND D. STRAWTHER
}

\begin{abstract}
A new characterization of strict convexity for complex normed linear spaces in terms of duality maps is given. It is then shown that many of the latest characterizations of strict convexity follow as simple corollaries.
\end{abstract}

1. Introduction. The concept of a strictly convex (s.c.) normed linear space has been extremely fertile. For example, strict convexity has proved useful in studies of the geometry of Banach spaces [5], [11], [18], orthogonality [11], semi-inner-products [1], [9], [10], [14], [15], [20] and nonlinear operators [6], [7], [17]. The most well-known characterizations of s.c. normed linear spaces are the following [8], [11], [18].

(1) If $\|x+y\|=\|x\|+\|y\|, x \neq 0$, then $y=c x$ for some $c \geqslant 0$.

(2) Every element of the unit sphere is an extreme point of the unit ball.

(3) Every nonzero continuous linear functional attains a maximum on at most one point of the unit sphere.

In this paper we give another characterization of s.c. normed linear spaces in terms of duality maps. This result can be used to unify and prove many of the other latest characterizations. For example, as simple corollaries of our result we obtain a characterization due to Menaker [15] (and mentioned by Palmer [16]), a characterization stated (but not proved) by Berkson [1], a characterization due to Torrance [20], a characterization half of which was proved by Husain and Malviya [10] and finally. a generalization of Petryshyn's characterization [7], [17], [19] to complex spaces.

2. A new characterization. Let $X$ be a complex normed linear space with unit sphere $S$. In this section we shall be concerned with a duality map $I$ : $X \rightarrow 2^{X^{*}}$ defined as follows:

$$
I(x)=\left\{f \in X^{*}: f(x)=\|f\|\|x\|\right\} .
$$

It is easy to see that $x=0$ if and only if $I(x)=X^{*}$. Also notice that for $c>0, I(x)=I(c x)=c I(x)$. We now give our main result.

THEOREM 1. The following statements are equivalent. (a) $X$ is s.c. (b) $I(y) \subseteq I(x)$ for $x \neq 0$ implies $y=c x$ for some $c>0$. (c) $I(y)=I(x)$ implies $y=c x$ for some $c \geqslant 0$.

Proof. (a) $\Rightarrow$ (b) Suppose $x \neq 0, I(y) \subseteq I(x)$ and $y \neq c x$ for some $c>0$. Now $y \neq 0$ since otherwise $I(x)=I(y)=X^{*}$ so $x=0$, which is a contradic-

Received by the editors May 2, 1975 and, in revised form, June 13, 1975.

AMS (MOS) subject classifications (1970). Primary 46B05, 46B10; Secondary 46C05, 47H05.

Key words and phrases. Strict convexity, normed linear spaces, Banach spaces, duality mappings, semi-inner-product spaces. 
tion. By the Hahn-Banach theorem there is an $f \in X^{*}$ such that $\|f\|=1$ and $f(y)=\|y\|$. Then $f \in I(y) \subseteq I(x)$. Now $y /\|y\| \neq x /\|x\|$ and $f(y /\|y\|)=$ $f(x /\|x\|)=\|f\|$. It follows from (3) that $X$ is not s.c. (b) $\Rightarrow$ (c) is trivial. (c) $\Rightarrow$ (a). Suppose $X$ is not s.c. Then by (2) there is a nontrivial line segment $L$ in $S$. Let $w, x, y, z$ be distinct elements of $L$ which satisfy $y=(x+w) / 2$, $x=(y+z) / 2$. Let $f \in I(y)$. Now $|f(x)| \leqslant\|f\|\|x\|=\|f\|$. Also

$$
|f(x)-2\|f\||=|f(x-2 y)|=|f(-w)| \leqslant\|f\|\|w\|=\|f\| .
$$

Hence $f(x)=\|f\|$ and $f \in I(x)$. Thus $I(y) \subseteq I(x)$ and, by similar reasoning, if $f \in I(x)$ then $f \in I(y)$ so $I(x)=I(y)$. Furthermore, $y \neq c x$ for some $c \geqslant 0$, since otherwise $w=d x$ for some real number $d$. Then, since $\|w\|=\|x\|$ $=1$ we would have $w= \pm x$. But since $w \neq x, w=-x$ and $y=0$ which is a contradiction.

3. Other characterizations. We now use Theorem 1 to prove other characterizations of strict convexity. Let $R^{+}$denote the nonnegative real numbers. A semipositive function $\phi$ is a map $\phi: R^{+} \rightarrow R^{+}$which satisfies $\phi(\lambda)=0$ if and only if $\lambda=0$. A pseudo-gauge function is a strictly increasing semipositive function. Pseudo-gauge functions generalize gauge functions as defined in [7] where, in addition, $\phi$ is required to be continuous and satisfy $\phi(\lambda) \rightarrow \infty$ as $\lambda \rightarrow \infty$. If $\phi$ is a semipositive function, we define a duality map $J_{\phi}: X \rightarrow 2^{X^{*}}$ as follows:

$$
J_{\phi}(x)=\left\{f \in X^{*}: f(x)=\|f\|\|x\|,\|f\|=\phi(\|x\|)\right\} .
$$

If $\phi$ is the function $\phi(\lambda)=\lambda$, we call $J_{\phi}$ the normalized duality map and denote it by $J$. The map $J$ was introduced by Beurling and Livingston [2] and studied by many others [3], [4], [6], [7], [12], [13], [17], [21]. Notice that if $\phi$ is a semipositive function, then $I(x)=\cup_{a \geqslant 0} a J_{\phi}(x)$ for all $x \in X$.

THEOREM 2. Let $\phi$ and $\psi$ be semipositive functions. If $X$ is s.c. then $J_{\phi}(x) \cap J_{\psi}(y) \neq \varnothing$ implies $y=c x$ for some $c>0$. If there exists $a>0$ such that $\phi(a)=\psi(a)$ and $J_{\psi}(y)=J_{\phi}(x)$ implies $y=c x$ for some $c \geqslant 0$, then $X$ is s.c.

Proof. Suppose $J_{\phi}(x) \cap J_{\psi}(y) \neq \varnothing$ and $y \neq c x$ for $c>0$. Let $f \in$ $J_{\phi}(x) \cap J_{\psi}(y)$. Now $f \neq 0$, since otherwise $x=y=0$. Also $x, y \neq 0$, since otherwise $\|f\|=\psi(\|y\|)=\phi(\|x\|)=0$. Furthermore, $y /\|y\| \neq x /\|x\|$ and $f(y /\|y\|)=f(x /\|x\|)=\|f\|$. By (3), $X$ is not s.c. Next, assume $\phi(a)=\psi(a)$ for some $a>0$ and $J_{\psi}(y)=J_{\phi}(x)$ implies $y=c x$ for some $c \geqslant 0$. Now suppose $I(y)=I(x)$. If either $x$ or $y$ is zero then so is the other. Suppose $x$, $y \neq 0$. If $f \in J_{\psi}(a y /\|y\|)$, then $f \in I(y)=I(x)=I(a x /\|x\|)$. Hence $\|f\|$ $=\psi(a)=\phi(a)=\phi(\|a x /\| x\|\|)$ so $f \in J_{\phi}(a x /\|x\|)$. Therefore, $J_{\psi}(a y /\|y\|)$ $\subseteq J_{\phi}(a x /\|x\|)$ and by symmetry these two sets are equal. Hence there exists a $c \geqslant 0$ such that $y=c x$. By Theorem $1, X$ is s.c.

Corollary 3. Let $\phi$ be a pseudo-gauge function. Then the following statements are equivalent. (a) $X$ is s.c. (b) $J_{\phi}(x) \cap J_{\phi}(y)=\varnothing$ whenever $x \neq y$. (c) $J_{\phi}(y) \subseteq J_{\phi}(x)$ whenever $x \neq y$. (d) $J_{\phi}(y) \neq J_{\phi}(x)$ whenever $x \neq y$. 
Proof. (a) $\Rightarrow$ (b) Suppose $X$ is s.c. and $J_{\phi}(x) \cap J_{\phi}(y) \neq \varnothing$. Then by Theorem 2,y $=c x$ with $c>0$. If $f \in J_{\phi}(x) \cap J_{\phi}(y)$ then $\phi(\|y\|)=\|f\|=\phi(\|x\|)$. If $x=0$, then $y=0$. If $x \neq 0$, since $\phi$ is injective, $\|y\|=\|x\|$. Hence $c=1$ and $x=y$. (b) $\Rightarrow$ (c) and (c) $\Rightarrow$ (d) are trivial. (d) $\Rightarrow$ (a) follows from Theorem 2 .

Corollary 4 (Menaker). $X$ is s.c. iff $J(x) \cap J(y)=\varnothing$ whenever $x \neq y$.

Corollary 5 (BerKSON). Let $[\cdot, \cdot]$ be a consistent semi-inner-product on $X$. Then $X$ is s.c. iff whenever $[x, y]=\|x\|\|y\|, x \neq 0$, then $y=c x$ for some $c \geqslant 0$.

Proof. Suppose $X$ is s.c. and $[x, y]=\|x\|\|y\|, x \neq 0$. Then

$$
(\|x\|+\|y\|)\|y\| \geqslant\|x+y\|\|y\| \geqslant[x+y, y]=(\|x\|+\|y\|)\|y\| .
$$

If $y=0$, we are finished; otherwise $\|x\|+\|y\|=\|x+y\|$. It follows from (1) that $y=c x$ for some $c \geqslant 0$. Conversely, suppose $X$ is not s.c. Then by Theorem 1 there are elements $x \neq 0, y \neq c x$, with $c>0$, such that $I(y)$ $\subseteq I(x)$. Since $y \neq 0, y \neq c x$ with $c \geqslant 0$. Let $f(z)=[z, y]$ for every $z \in X$. Then $f \in J(y)$ and hence $f \in I(x)$. Thus $[x, y]=f(x)=\|f\|\|x\|=\|x\|\|y\|$.

COROLlary 6 (TORRANCE). Let $[\cdot, \cdot]$ be a consistent semi-inner-product on $X$. Then $X$ is s.c. iff whenever $\|y+z\| \leqslant\|y\|$ and $[z, y]=0$, then $z=0$.

Proof. The corollary is equivalent to the following more convenient statement. $X$ is s.c. iff whenever $[x, y]=\|y\|^{2}, x \neq y$, then $\|x\|>\|y\|$. Suppose $X$ is s.c., $x \neq y$ and $[x, y]=\|y\|^{2}$. If $\|x\|=\|y\|$, then by Corollary 5, $y=c x$, $c \geqslant 0$ which implies $x=y$, a contradiction. But $\|x\|\|y\| \geqslant[x, y]=\|y\|^{2}$ so $\|x\|>\|y\|$. The converse proceeds as in Corollary 5 .

Let $[\cdot, \cdot]$ be a consistent semi-inner-product on $X$. We say that a sequence $x_{n}$ converges weakly in the second argument to $x$ if for any $y \in X,\left[y, x_{n}\right] \rightarrow[y$, $x$ ] as $n \rightarrow \infty$. Husain and Malviya [10] have shown that if $X$ is s.c. then weak limits in the second argument are unique. We next show that a converse holds, thus giving another characterization of strict convexity.

COROLlary 7. $X$ is s.c. iff weak limits in the second argument are unique for every consistent semi-inner-product on $X$.

Proof. This corollary is equivalent to the following statement. $X$ is s.c. iff for every consistent semi-inner-product $[z, x]=[z, y]$ for every $z \in X$ implies $x=y$. Suppose $X$ is s.c. and $[z, x]=[z, y] \equiv f(z)$ for all $z \in X$. Then $f \in J(x) \cap J(y)$ and by Corollary $4, x=y$. Conversely, if $X$ is not s.c., then by Corollary 4, there exist $x \neq y$ and an $f \in J(x) \cap J(y)$. Define a consistent semi-inner-product as follows: $[u, v]=f_{v}(u)$ where $f_{v} \in J(v), v \neq x, y$, $f_{x}=f_{y}=f$. Then for every $z \in X,[z, x]=f_{x}(z)=f_{y}(z)=[z, y]$.

Let $\phi$ be a semipositive function. We say that $J_{\phi}$ is strictly monotone if for every $x \neq y$ and every $f \in J_{\phi}(x), g \in J_{\phi}(y)$ we have $\operatorname{Re}(f-g)(x-y)>0$. This generalizes the usual concept of strict monotonicity in real spaces which has been extensively studied in the literature [3], [7], [17], [19], [21]. Our next result generalizes Petryshyn's characterization [7], [17], [19] to complex spaces. 
COROllary 8. Let $\phi$ be a pseudo-gauge function. Then $X$ is s.c. iff $J_{\phi}$ is strictly monotone.

Proof. If $f \in J_{\phi}(x), g \in J_{\phi}(y)$, then the following identity holds:

$$
\begin{aligned}
\operatorname{Re}(f-g)(x-y)= & \|f\|\|x\|-\operatorname{Re} f(y)-\operatorname{Re} g(x)+\|g\|\|y\| \\
= & {[(\|f\|-\|g\|)(\|x\|-\|y\|)]+[\|f\|\|y\|-\operatorname{Re} f(y)] } \\
& +[\|g\|\|x\|-\operatorname{Re} g(x)] .
\end{aligned}
$$

Since each term in square brackets is nonnegative, $\operatorname{Re}(f-g)(x-y) \geqslant 0$ and $\operatorname{Re}(f-g)(x-y)=0$ iff $\operatorname{Re} f(y)=\|f\|\|y\|, \operatorname{Re} g(x)=\|g\|\|x\|$ and $(\|f\|-\|g\|)(\|x\|-\|y\|)=0$. If $\operatorname{Re} f(y)=\|f\|\|y\|$ then

$$
[\operatorname{Im} f(y)]^{2}+\|f\|^{2}\|y\|^{2}=[\operatorname{Im} f(y)]^{2}+[\operatorname{Re} f(y)]^{2}=|f(y)|^{2} \leqslant\|f\|^{2}\|y\|^{2} .
$$

It follows that $\operatorname{Re} f(y)=\|f\|\|y\|$ iff $f(y)=\|f\|\|y\|$ and, similarly, $\operatorname{Re} g(x)=\|g\|\|x\|$ iff $g(x)=\|g\|\|x\|$. Furthermore, if $\|f\|=\|g\|$, then $\phi(\|x\|)$ $=\|f\|=\|g\|=\phi(\|y\|)$ and, since $\phi$ is strictly increasing, $\|x\|=\|y\|$. Hence $(\|f\|-\|g\|)(\|x\|-\|y\|)=0$ iff $\|f\|=\|g\|$ and $\|x\|=\|y\|$. Using these facts and Corollary 3 we obtain: $X$ is not s.c. iff there exists $x \neq y$ and an $f \in X^{*}$ such that $f \in J_{\phi}(x) \cap J_{\phi}(y)$ iff $f(x)=\|f\|\|x\|, f(y)=\|f\|\|y\|,\|f\|=\phi(\|x\|)$ $=\phi(\|y\|), x \neq y$ iff there exists $x \neq y, f \in J_{\phi}(x), g \in J_{\phi}(y)$ with $\operatorname{Re}(f-g)$ $(x-y)=0$ iff $J_{\phi}$ is not strictly monotone.

\section{REFERENCES}

1. E. Berkson, Some types of Banach spaces, Hermitian operators, and Bade functionals, Trans. Amer. Math. Soc. 116(1965), 376-385. MR \#4554.

2. A. Beurling and A. E. Livingston, $A$ theorem on duality mappings in Banach spaces, Ark. Mat. 4(1962), 405-411. MR 26 \#2851.

3. F. Browder, Multi-valued monotone nonlinear mappings and duality mappings in Banach spaces, Trans. Amer. Math. Soc. 118(1965), 338-351. MR 31 \#5114.

4. __ On a theorem of Beurling and Livingston, Canad. J. Math. 17(1965), 367-372. MR 31 \# 595

5. J. Clarkson, Uniformly convex spaces, Trans. Amer. Math. Soc. 40(1936), 396-414.

6. D. deFigueiredo, Topics in nonlinear functional analysis, Lecture Notes, \#48, University of Maryland, 1967.

7. C. R. DePrima and W. V. Petryshyn, Remarks on strict monotonicity and surjectivity properties of duality mappings defined on real normed linear spaces, Math. Z. 123(1971), 49-55. MR 46 \# 7977.

8. N. Dunford and J. T. Schwartz, Linear operators. 1: General theory, Pure and Appl. Math., vol. 7, Interscience, New York, 1958. MR 22 \#302.

9. J. Giles, Classes of semi-inner-product spaces, Trans. Amer. Math. Soc. 129(1967), 436-446. MR 36 \#663.

10. T. Husain and B. Malviya, On semi-inner-product spaces. I, Colloq. Math. 24(1972), 235-239. MR 47 \#9255.

11. R. C. James, Orthogonality and linear functionals in normed linear spaces, Trans. Amer. Math. Soc. 61(1947), 265-292. MR 9, 42.

12. T. Kato, Nonlinear semigroups and evolution equations, J. Math. Soc. Japan 19(1967), 508-520. MR 37 \#1820.

13. J.-L. Lions, Quelques méthodes de résolution de problèmes aux limites non linéaires, Dunod; Gauthier-Villars, Paris, 1969. MR 41 \# 4326.

14. G. Lumer, Semi-inner-product spaces, Trans. Amer. Math. Soc. 100(1961), 29-43. MR 24 \# A2860. 
15. M. Menaker, Semi-inner-product spaces, Thesis, University of Maryland, 1969.

16. T. Palmer, Unbounded normal operators on Banach spaces, MRC Technical Summary Report \#785, 1967.

17. W. Petryshyn, A characterization of strict convexity of Banach spaces and other uses of duality mappings, J. Functional Analysis 6(1970), 282-291. MR 44 \#4496.

18. V. Smul'jan, On some geometrical properties of the unit sphere in spaces of the type (B), Mat. Sb. 48(1938), 90-94.

19. D. Strawther and S. Gudder, A characterization of strictly convex Banach spaces, Proc. Amer. Math. Soc. 47(1975), 268.

20. E. Torrance, Strictly convex spaces via semi-inner-product space orthogonality, Proc. Amer. Math. Soc. 26(1970), 108-110. MR 41 \#5943.

21. M. M. Vaĭnberg, Equations with nonlinear accretive and monotone operators, and GalerkinPetrov approximations, Dokl. Akad. Nauk SSSR 197(1971), 754-757 = Soviet Math. Dokl. 12(1971), 525-529. MR 43 \# 7985.

Department of Mathematics, University of Denver, Denver, Colorado 80210 (Current address of D. Strawther)

Current address (S. P. Gudder): Department of Mathematics, University of Nottingham, Nottingham NG7 2RD, England 